antiphospholipid biology without APS, Prothrombin Time $<50 \%$, activated Partial Thromboplastin Time ratio $>1.2$, platelets $<50 \mathrm{G} / \mathrm{L}$ and biopsied kidney size were not associated with a higher rate of complications.

The number of glomeruli sampled and the performance of the biopsy to establish a histologic diagnostic were similar in the 4 groups.

Conclusions: TJRB provides diagnostic yield and safety similar to those of TCRB. It should be considered as a first intention procedure for SLE and APS patients at risk of bleeding.

Disclosure of Interest: None declared

DOI: 10.1136/annrheumdis-2018-eular.1645

\section{SAT0437 INITIAL MANIFESTATIONS OF CHILDHOOD-ONSET SYSTEMIC LUPUS ERYTHEMATOSUS: RESULTS OF SINGLE CENTRE RETROSPECTIVE STUDY}

M.I. Kaleda, E.A. Aseeva, I.P. Nikishina, S.K. Soloviev. Pediatrics, V.A. Nasonova Research Institute of Rheumatology, Moscow, Russian Federation

Background: Approximately $10 \%-20 \%$ of patients (pts) with SLE develop lupus before 18 years old. Childhood-onset SLE (cSLE) usually has more aggressive course. The achievement of medication-free remission in adulthood is extremely rare in cSLE and quality of life remains compromised.

Objectives: To establish the specific features of cSLE at disease onset by the retrospective study in single centre.

Methods: 216 pts with cSLE who were hospitalised in our centre from 1992 to 2017 were included in retrospective study. Diagnosis of SLE was reviewed according to 2012 SLICC criteria. Clinical, haematological and immunological manifestations of SLE were evaluated. SLEDAI $2 \mathrm{~K}$ was used for disease activity assessment.

Results: $12.9 \%$ of cSLE pts were boys (girls to boys ratio was $6.7: 1$ ). The triggering factor was found in $29 \%$ pts: solar exposure - in $51 \%$, previous infection - in $19 \%$, stressful situation - in $12 \%$, vaccination - in 10\%, menarche - in 5\%, high physical activity - in 3\%. Only $9.2 \%$ of patients initially had SLE diagnosis, $1.4 \%$ had discoid lupus erythematosus as an initial diagnosis, $21 \%$ - different infections, $11.2 \%$ - allergic diseases, $6.3 \%$ - nephritis, $33.6 \%$ - various rheumatic diseases $(16.8 \%$ - juvenile idiopathic arthritis), in remaining $17.3 \%$ cases the information about initial diagnosis was missing. The median age at the onset was 13.7 y $(10.8 ; 15.05)$; the median disease duration at the time of cSLE verification was 6 months. ${ }^{2 ;} 14$ In $33.4 \%$ pts cSLE was verified after 1 year disease duration, in $15.3 \%$ - after 3 years. The most common feature was arthritis - in $71.4 \%$. Fever observed in $68.5 \%$ pts at the onset, significant weight loss - in $29.4 \%$. $64 \%$ pts had acute cutaneous lupus at the onset, $42.4 \%$ - chronic cutaneous lupus, $17.7 \%$ - oral and nasal ulcers, $22.2 \%$ - nonscarring alopecia, 31\% - serositis, 56.6\% renal involvement, $21.2 \%$-neuropsychiatric disorder. The Coombs' positive hemolytic anaemia was found in $15.8 \%$ pts, leucopenia/lymphopenia - in $52.2 \%$, thrombocytopenia - in $23.6 \%$. ANA were detected in $100 \%$ pts, anti-dsDNA - in $83.3 \%$, anti-Sm - in $29.2 \%$, antiphospholipid antibodies - in $7.3 \%$, hypocomplementemia - in $49.0 \%$, positive direct Coombs test out of hemolytic anaemia - in $13,5 \%$. Macrophage activation syndrome at the onset was documented in $3.4 \%$ pts. Median disease activity by SLEDAI at the time of CSLE verification was 13.7 scores, ${ }^{8 ; 20}$ maximum -42 .

Conclusions: cSLE presentation with non-specific general and constitutional manifestations in the majority of cases misled to erroneous interpretation of the condition as infectious or allergic disease in $1 / 3$ of all cases. A monosymptomatic manifestation at the onset, such as arthritis, skin lesion or hematologic disorders, can lead to late diagnosis and very high activity at the moment of start therapy. Specific features of cSLE must be suspected in all cases of arthritis with skin lesions and/or any haematological manifestations, even non-specific.

Disclosure of Interest: None declared

DOI: 10.1136/annrheumdis-2018-eular.3683

\section{SAT0438 INCREASED RISK OF DEPRESSION IN PATIENTS WITH CUTANEOUS LUPUS ERYTHEMATOSUS AND SYSTEMIC LUPUS ERYTHEMATOSUS: A DANISH NATIONWIDE COHORT STUDY}

J.H. Hesselvig ${ }^{1}$, A. Egeberg ${ }^{1}$, K. Kofoed ${ }^{1}$, G. Gislason ${ }^{2}$, L. Dreyer ${ }^{3}$. ${ }^{1}$ Department of Dermatology and Allergy; ${ }^{2}$ Department of Cardiology; ${ }^{3}$ Department of Rheumatology, Herlev and Gentofte University Hospital, Copenhagen, Denmark

Background: Quality of life is considerably impaired in patients with systemic lupus erythematosus (SLE) as well as in patients with cutaneous lupus erythematosus (CLE). In patients with SLE, prevalence estimates of depression ranges considerably[, ${ }^{1}$ while the prevalence of depression in CLE remains severely understudied. Data on the actual risk of depression in adults after being diagnosed with CLE or SLE remain scarce.
Objectives: To examine whether patients with SLE or CLE have increased risk of depression.

Methods: In this nationwide observational cohort study, we included patients $\geq 18$ years with a first-time diagnosis of SLE or CLE between 2000-2015 identified in the Danish National Patient Register matched with people from the general population in Denmark in a 1:10 ratio. After linkage to various national Danish health registers analyses of risk for depression and antidepressant use were performed in Cox regression models adjusted for age, sex, socio-economic status, smoking, alcohol abuse, prior depression, and prior antidepressant use.

Results: A total of 3489 patients with lupus erythematosus and 34890 people from the general population were included. The adjusted hazard ratios (HRs) of depression were $1.87(95 \% \mathrm{Cl}, 1.37-2.55)$ and $2.15(95 \% \mathrm{Cl}, 1.66-2.77)$ fo patients with CLE and SLE, respectively, compared to the general population. The adjusted HRs of antidepressant use were $1.36(95 \% \mathrm{Cl} 1.22-1.51)$ and 1.76 (95\% Cl 1.62-1.91) for patients with CLE and SLE, respectively. The risk of depression was more pronounced in patients diagnosed $<50$ years of age; adjusted $\mathrm{HR}=2.88(95 \% \mathrm{Cl}, 1.77-4.69)$ for $\mathrm{CLE}$ and $\mathrm{HR}=2.33(95 \% \mathrm{Cl}, 1.56-$ 3.49) for SLE. Also a high risk of depression was observed for men with CLE (HR $2.59,95 \% \mathrm{Cl}, 1.24-5.42)$ and SLE (HR 2.23, 95\% Cl, 1.11-4.48). Neither CLE or SLE were associated with increased risk of death from suicide.

Abstract SAT0438 - Table 1. Crude and adjusted hazard ratios for depression and antidepressant use in patients with lupus erythematosus compared with the general population

\begin{tabular}{|c|c|c|c|c|c|c|}
\hline & $\begin{array}{c}\text { Crude } \\
\text { HR }\end{array}$ & (95\% Cl) & P-value & $\begin{array}{c}\text { Adjusted } \\
\text { HR }^{\star}\end{array}$ & $(95 \% \mathrm{Cl})$ & P-value \\
\hline \multicolumn{7}{|c|}{ Depression } \\
\hline CLE & 2.34 & $\begin{array}{c}(1.71- \\
3.18)\end{array}$ & $<0.0001$ & 1.87 & $\begin{array}{c}(1.37- \\
2.55)\end{array}$ & 0.0001 \\
\hline SLE & 2.33 & $\begin{array}{c}(1.81- \\
3.01)\end{array}$ & $<0.0001$ & 2.15 & $\begin{array}{c}(1.66- \\
2.77)\end{array}$ & $<0.0001$ \\
\hline
\end{tabular}

Antidepressant

use

CLE

SLE

$\begin{array}{cccccc}1.61 & (1.45- & <0.0001 & 1.36 & (1.22- & <0.0001 \\ & 1.79) & & & 1.51) & \\ 1.94 & (1.78- & <0.0001 & 1.76 & (1.62- & <0.0001 \\ & 2.10) & & & 1.91) & \end{array}$

* Adjusted for age, sex, smoking, alcohol abuse, socio-economic status, prior depression, and prior antidepressant use.

$\mathrm{Cl}$, confidence interval; CLE, cutaneous lupus erythematosus; HR, Hazard Ratio; SLE, systemic lupus erythematosus.

Conclusions: The risk of depression was significantly increased in patients with SLE or CLE. We recommend that rheumatologists and dermatologists are aware of the increased risk of depression in patients with SLE or CLE.

\section{REFERENCE:}

[1] Zhang L, Fu T, Yin R, et al. Prevalence of depression and anxiety in systemic lupus erythematosus: a systematic review and meta-analysis. BMC Psychiatry 2017;17:70. doi:10.1186/s12888-017-1234-1

Disclosure of Interest: None declared

DOI: 10.1136/annrheumdis-2018-eular.2874

\section{SAT0439 DIFFERENTIAL LEVELS OF NOVEL AND CLASSIC ANTIPHOSPHOLIPID ANTIBODIES AMONG PATIENTS WITH PRIMARY ANTIPHOSPHOLIPID SYNDROME AND SYSTEMIC LUPUS ERYTHEMATOSUS}

J.A. Gómez-Puerta ${ }^{1,2}$, T. Urrego ${ }^{3}$, A. Hernández ${ }^{4}$, S. Ruiz ${ }^{4}$, S.M. Osorno ${ }^{2}$, C. Rua ${ }^{4}$, J. Duque Botero ${ }^{5}$, A.L. Vanegas-García ${ }^{6,7}$, C.H. Muñoz ${ }^{6,7}$, L. A. González ${ }^{6}, \mathrm{G}$. Vásquez ${ }^{3,6}$. ${ }^{1}$ Rheumatology, Hospital Clínic, Barcelona, Spain; ${ }^{2}$ Dinámica IPS; ${ }^{3}$ Grupo de Inmunología Celular e Inmunogenética; ${ }^{4}$ Medicina Interna; ${ }^{5}$ Grupo de Investigación en Trombosis; ${ }^{6}$ Grupo de Reumatología, Universidad de Antioquia; ${ }^{7}$ Servicio de Reumatología, Hospital San Vicente Fundación, Medellín, Colombia

Background: High levels of antiphospholipid antibodies (aPL) are associated predominantly with a higher risk of thrombosis, however, information about differential levels according underlying diagnosis is less well known.

Objectives: Our aim was to compared serum levels of 2 novel aPL (anti-phos phatidylserine/prothrombin (PS/PT) antibodies and anti domain 1 against B2 gly coprotein I (anti-D1 B2GP1)) and "classic" (anticardiolipin, aCL and anti B2GP1 antibodies) among patients with primary APS, SLE with and without thrombosis Methods: In this cross-sectional study, Anti-D1 B2GPI antibodies were tested using a chemiluminescent immunoassay (QUANTA Flash, Inova Diagnostics). In 